\title{
EM Induction in the New Zealand Southern Alps Region
}

\author{
H. W. Dosso ${ }^{1}$, J. CHEN ${ }^{1}$, and C. J. BRomLeY ${ }^{2}$ \\ ${ }^{1}$ Department of Physics and Astronomy, University of Victoria, Victoria, B.C., V8W 3P6, Canada \\ ${ }^{2}$ Institute of Geological \& Nuclear Sciences, Private Bag 2000 Taupo, New Zealand
}

(Received May 22, 1995; Revised August 21, 1995; Accepted October 24, 1995)

\begin{abstract}
The geomagnetic coast effect is known to comprise a major component in magnetic field observations at coastal sites. As the New Zealand South Island is relatively narrow, significant induction effects of the surrounding oceans are expected at most on-land sites. Interpretations of the observations as the responses of anomalous conductors would be simplified if these coast effects were not present. In this work, the removal of the coast effects at sites on the South Island is accomplished by subtracting analogue model coast effect induction arrows from the field site induction arrows. With the coast effects removed, the resulting difference induction arrows at some of the 26 sites would appear to be attributable to the induction responses of some of the sedimentary basins and faults, structures that were not included in the analogue model simulations.
\end{abstract}

\section{Introduction}

Geomagnetic field observations in New Zealand, where sites can nowhere be far from the surrounding ocean, are expected to be strongly affected by the electromagnetic responses of the ocean. These ocean responses, known as coast effects (commonly expressed in the form of in-phase and quadrature induction arrows), when combined with the responses of anomalous conductors (e.g. conductive faults, sedimentary basins), lead to difficulties in the interpretation of geomagnetic observations. Clearly, the difficulties would be reduced if the coast effects were subtracted before interpretations were attempted. In order that the difference induction arrows (residuals after such a subtraction) be the actual responses of the anomalous conductor, accurate coast effect values would be required.

The limitations to such a subtraction of the coast effect have been examined by Wolf (1983), Weaver and Agarwal (1991), and Dosso and Meng (1992) using numerical model calculations, and by Chen (1994) using laboratory measurements for analogue models of conductive faults parallel to a nearby ocean coastline. This latter work demonstrated that except for locations right at the coastline, the coast effect response for a model ocean could be subtracted with reasonable accuracy (to within a few percent) from measured responses of models that included both the ocean and an elongated conductor parallel to the coastline, to yield the responses of the conductor (fault) alone. This has particular application to geomagnetic studies in New Zealand where the faults in most regions are roughly parallel to the coastlines. The analogue model study of the responses of a range of elongated conductors both parallel and perpendicular to the coastline, and the removal of the coast effects is the subject of a future work.

Bapat et al. (1993) and Chamalaun and McKnight (1993) subtracted coast effects, that were numerically calculated for thin sheet models, from field site induction arrows to obtain the difference induction arrows at sites in Japan and New Zealand respectively. In both studies the difficulty of including adequate spatial detail of the complex coastlines and the ocean bathymetry limited the applicability of the calculated coast effects. The conclusions in each case were that the ocean responses dominated the observed induction arrow responses, and that more accurate coast effect values were needed to permit fully separating these ocean effects from the inductive responses of any underlying anomalous conductors.

Carefully constructed laboratory analogue models of coastal regions do physically permit more accurate simulations of the complex coastline contours and the very important ocean bathymetry than has 
to date been accomplished in numerical models. Meng and Dosso (1990) successfully employed laboratory analogue model coast effects to subtract the ocean induction responses from field observations to yield responses of anomalous conductors at depth in the Japan region. More recently, Kang et al. (1993) and Chen et al. (1993) have also used analogue model measurements to determine the coast effects at 11 sites in southwest Nigeria and at the New Zealand Eyrewell Observatory respectively. In both cases the resulting in-phase and quadrature difference arrows were interpreted as the responses of anomalous conductors (such as conductive faults and sediments) in the survey regions.

Expressing the geomagnetic responses as the well-known in-phase and quadrature induction arrows is convenient for the purpose of interpreting geomagnetic field results since the arrow directions give information on the locations of induced current concentrations in conductive bodies, and the arrow lengths provide some indication of the proximity and the spatial extent of the anomalous conductors. For single conductors large in-phase arrows tend to indicate current concentrations nearby and at shallow depths, while quadrature arrow responses that increase with period imply current induced at larger depths.

In the present work, analogue model coast effect induction arrows from the New Zealand analogue model study of Chen et al. (1993) are subtracted from the geomagnetic induction arrows of Bromley (1979) at 26 field sites in the New Zealand Southern Alps region. With the coast effects removed, an attempt is made to interpret qualitatively the resulting difference arrows at a given site as the resultant response of induction in any anomalous conductors present in the region but not included in the analogue model simulation. Anomalous conductors in the Southern Alps region would be sedimentary basins and those faults with significant fluid and metallic mineral content.

\section{Model and Field Site Induction Arrow Responses}

The scaled laboratory analogue model of the New Zealand region has been discussed in detail previously (Chen et al., 1990, 1993). Suffice it to say here that a graphite plate $\left(1.4 \times 1.4 \mathrm{~m}^{2}\right)$ that simulated a $2800 \mathrm{~km} \times 2800 \mathrm{~km}$ area ocean surrounding the two islands was shaped according to the scaled island coastline contours and precisely machined to simulate the ocean bathymetry. This graphite model ocean, suspended at the surface of the concentrated brine solution in a large wooden tank, then provided a simulation of New Zealand and the surrounding ocean for the purpose of studying magnetic induction coast effects.

Measurements of the in-phase and quadrature magnetic field components $\left(B_{x}, B_{y}, B_{z}\right)$ were carried out for a range of periods along a detailed grid of traverses over the model for two perpendicular inducing source field polarizations. For each polarization the source field was held constant for all measurements at a common remote reference location. The measurements were used to calculate the model in-phase and quadrature coast effect induction arrows for points along each traverse. In the present work, time-varying fields of the form $e^{i \omega t}$ were used and the signs of both induction arrow components reversed following the convention of Lilley and Arora (1982), so that the induction arrows would point towards (rather than away from) current concentrations.

Bromley (1979) carried out geomagnetic depth sounding measurements and obtained induction arrows for short periods (up to $10 \mathrm{~min}$ ) at 26 sites in the Southern Alps region of South Island. To demonstrate the differences between the observed geomagnetic induction arrows (dashed arrows) obtained by Bromley (1979) and the model coast effect induction arrows (solid arrows) obtained by Chen et al. (1993), both field site and model arrows at each of the 26 sites at $10 \mathrm{~min}$ period for the in-phase component are shown in Fig. 1. (Note that arrow heads are not shown in cases where the induction arrows are very short). Included in this figure are the code names of the field sites, the locations of the sedimentary basins (S1-S5), and the numerous faults as provided in the earlier work of Bromley (1979). The model arrows in the central regions are very short as expected for essentially a $2 \mathrm{D}$ island, since in traversing this relatively narrow island, the coast effect responses must reverse sign, and thus be near zero over a considerable area in the central region. Thus in this region, the geomagnetic induction arrows should be primarily the responses of local anomalous conductors. The coast effect responses at short periods are 


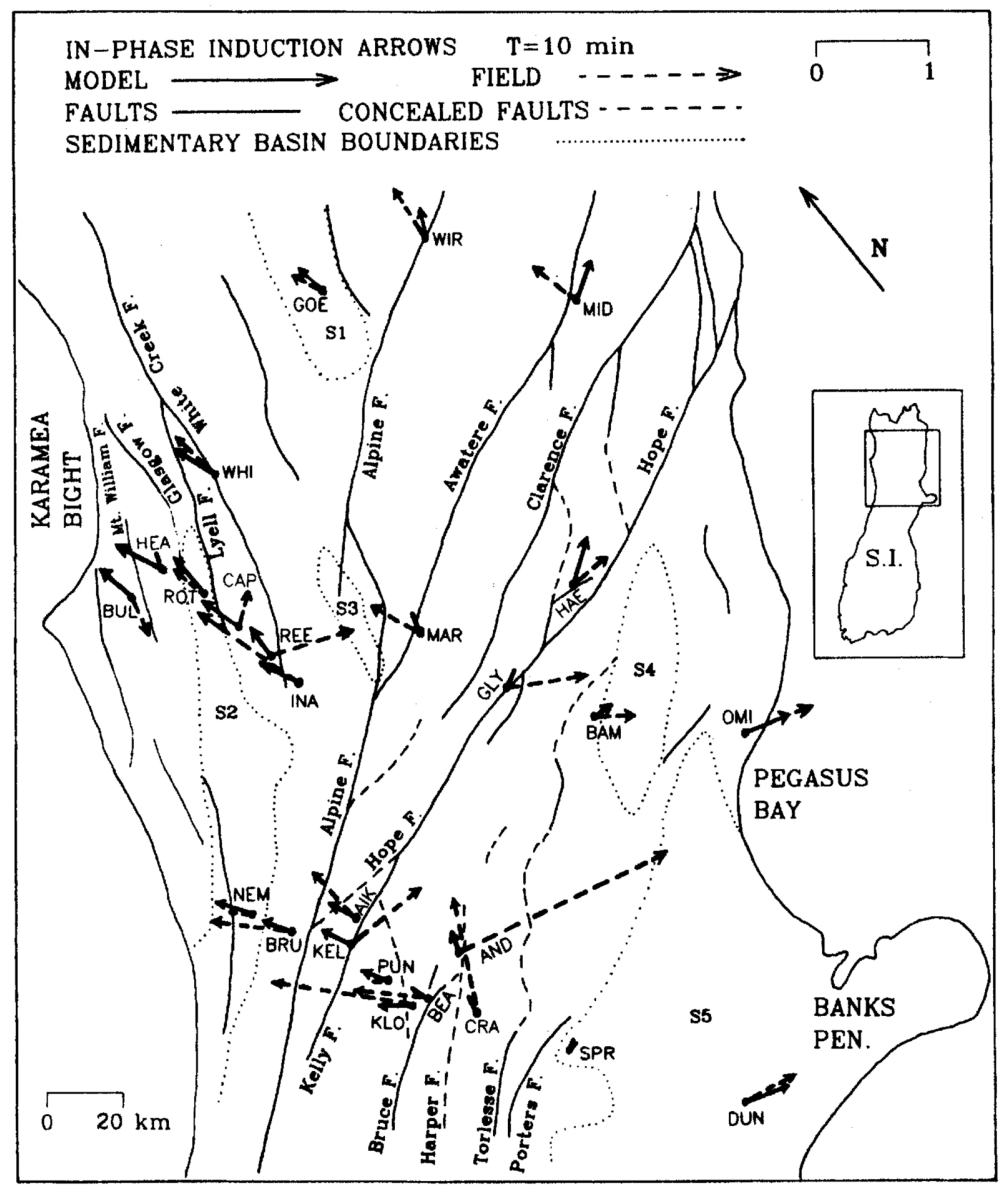

Fig. 1. The analogue model (Chen et al., 1993) and field site induction arrows at 26 sites (Bromley, 1979) in the New Zealand South Island Southern Alps region.

known generally to be much smaller for the quadrature component than for the in-phase component. This is certainly the case for the model coast effects at $10 \mathrm{~min}$, with the quadrature arrows, even right at the coast, being very short. Thus, any significant quadrature arrows would be due to anomalous conductors, rather than the coast effect.

At a number of sites in Fig. 1, both near the coast and inland, the model and field site arrows are seen to differ considerably both in magnitude and direction. For example, at BUL (near Karamea Bight) the model in-phase arrow points seaward while the field arrow points landward. The arrows at REE also are roughly oppositely directed, with significant differences in magnitude. At sites where the model and field site arrows are in close agreement, for example at DUN (lower right), the field site arrows are then primarily the geomagnetic coast effect arrows, since it is these arrows alone that the analogue model arrows simulate. In the present work, an appropriate site for checking the validity of such a subtraction would be this site (DUN), about $20 \mathrm{~km}$ from the coast (near Banks Peninsula). With no nearby anomalous conductors, other than the ocean, the model and geomagnetic (field) arrows should be approximately the same in magnitude and direction. This is certainly the seen to be the case for the in-phase components in Fig. 1. Subtracting the model arrow (coast effect responses) from the geomagnetic arrow clearly yields a roughly zero response, as it should be for a valid subtraction of the coast effect. This is also the case for the quadrature components at this site (not shown), being almost identical in magnitude ( 0.1 each) and 
direction (pointing eastward). Assuming the model coast effect arrows at the other sites on this island to be roughly true representations of the actual coast effects, a difference in the geomagnetic and the model arrows at a given site should be indicative of the presence of an anomalous conductor, a conductor not simulated in the analogue model.

It is realized that errors in the model and the field arrows will limit the accuracy of the difference arrows, and hence the reliability of the interpretation. While the statistical errors in the model arrows were very small (measurements repeatable to within less than 1\%), this was not the case for the geomagnetic arrows. Taking into account the fact that the noise level in the field measurements at some sites was reported (Bromley, 1979) as being as large as, or even larger than, the signal itself, it is proposed that difference arrows of magnitude less than 0.2 be considered too small to warrant much consideration in any interpretation. Field sites where such large noise levels in the measurements occurred will be noted in the discussion of the induction arrow responses.

\section{Interpretation of Difference Induction Arrow Responses}

Figures 2(a) and 2(b) respectively show the in-phase and quadrature difference induction arrows for

(a)

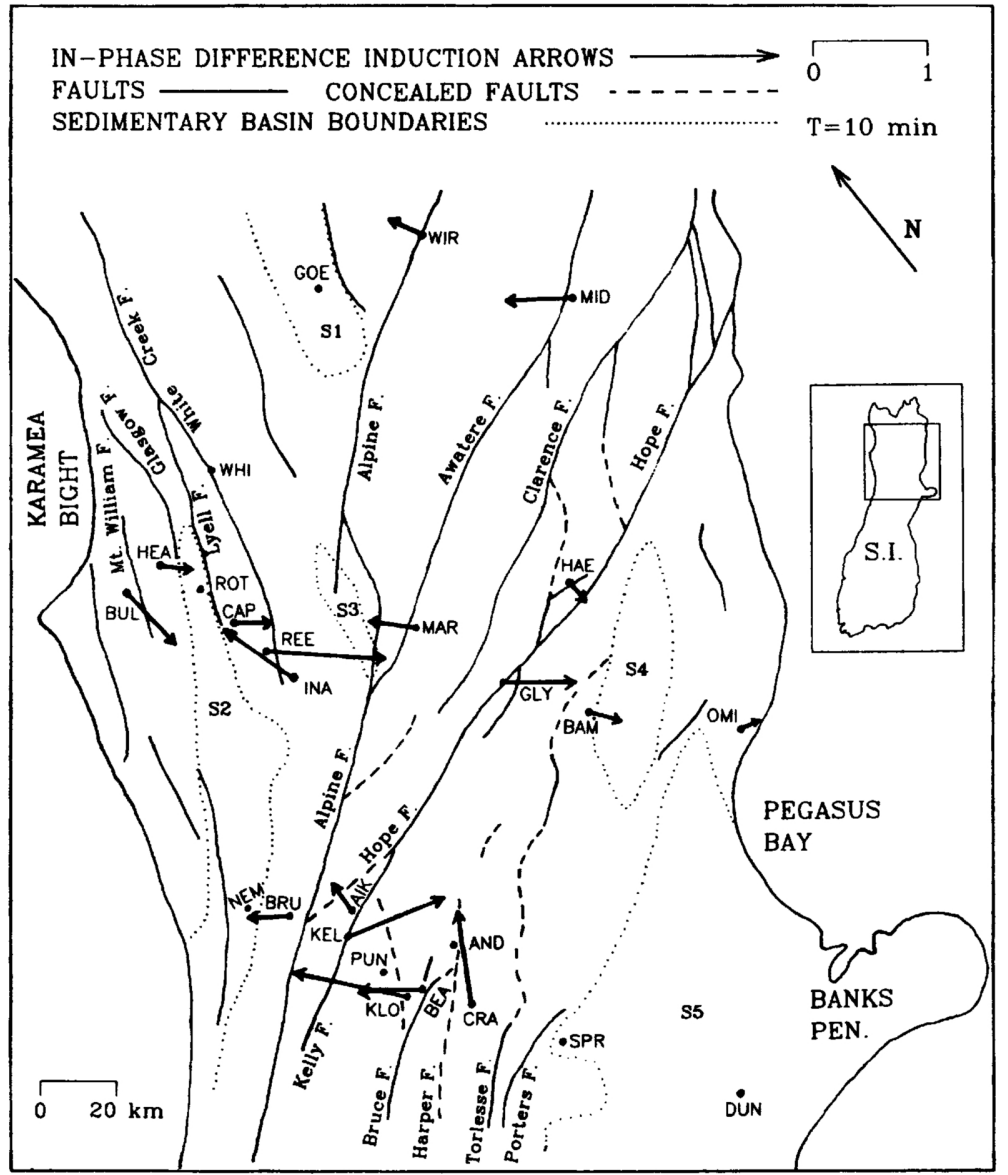

Fig. 2. (a) The in-phase difference induction arrows at the 26 sites (Bromley, 1979) in the New Zealand South Island Southern Alps region. (b) The quadrature difference induction arrows at the 26 sites (Bromley, 1979) in the New Zealand South Island Southern Alps region. 
10 min period at 26 sites in the Southern Alps region of New Zealand. Geomagnetic quadrature arrows were not available at CAP, MID, HAE and HEA, and thus quadrature difference arrows are not given at these sites. At some other sites (GEO, ROT, NEM, PUN, SPR, DUN in Fig. 2(a); and AIK, BAM, CRA, SPR, DUN in Fig. 2(b)) no arrows are shown since the ocean effect subtraction resulted in a near zero residual response.

\subsection{Sites over sedimentary basins}

With the coast effect responses removed, the in-phase arrow responses at the sites directly over the sedimentary basins (sites GOE over basin S1; ROT and NEM over S2; and SPR and DUN over S5) are seen to be negligible, as would be expected, due to the attenuation effects of the underlying conductive basins. Differing from the in-phase arrows, the quadrature arrows at GOE and NEM, do show some responses at this $10 \mathrm{~min}$ period. This could be expected, if the sites were near anomalous conductors that were substantially deeper than the shallow sedimentary basins. Nearby faults that are conductive at large depths would be examples of such anomalous conductors. The quadrature arrow at GOE and NEM pointing south-eastward, could indicate induction at depth in the Alpine Fault, assuming this fault to be conductive. The quadrature arrow at ROT (upper section of S2) shows too small a response to warrant consideration. At SPR and DUN (lower right in Figs. 2(a) and 2(b)), the negligible in-phase and quadrature

(b)

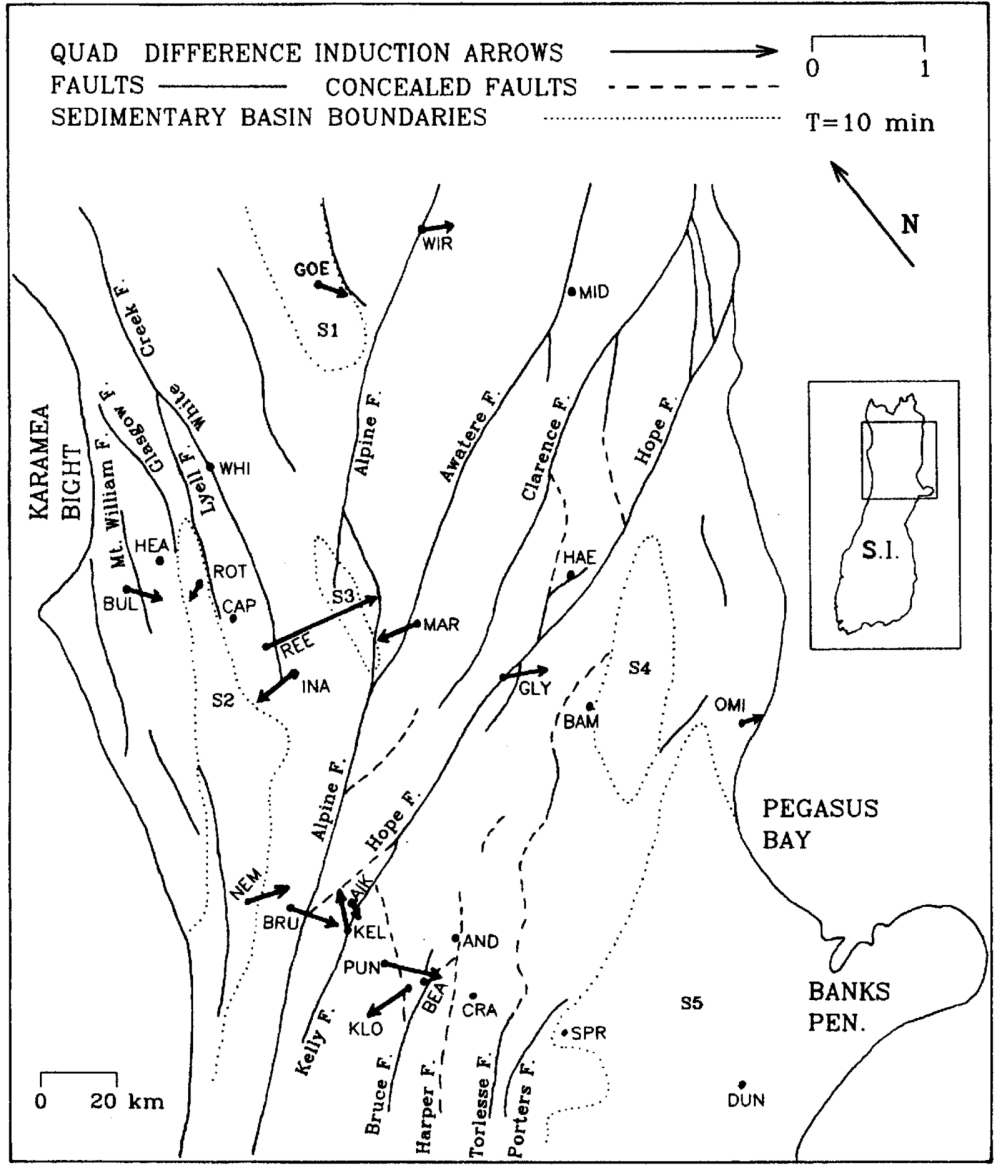

Fig. 2. (continued). 
responses would be consistent with no nearby major anomalous conductors, other than the ocean, the response of which has been subtracted.

\subsection{Sites near sedimentary basins}

The in-phase and quadrature induction arrows at a site near a conductive basin should include components pointing towards the basin. However, in this complex faulted Southern Alps region, these induction arrows at a given site will normally be the resultant of the responses of multiple conductors (e.g. sedimentary basins and faults, if conductive), and thus at a site even very near the basin boundary, the arrows may not necessarily point towards the basin. Further, since the in-phase and quadrature responses at a given site between conductors have different period dependences, one arrow may point towards the basin, while the other points away from the basin (towards another conductor) at the same given period.

At HEA (the site just to the west of the northern end of basin S2) the in-phase arrow in Fig. 2(a) points south-eastward towards the basin as would be expected in the absence of larger oppositely directed responses of any other nearby anomalous conductors to the northwest. At this site, however, if the sum of the responses of the S2 basin and the nearby Lyell and White Creek Faults (if conductive), all to the southeast, is greater than any oppositely directed response of the Mt. William Fault (if conductive) to the northwest, the resultant arrow would have the direction shown in Fig. 2(a). At this site, however, the noise level in the field measurements was particularly large, and thus the in-phase arrow would have rather limited validity. The quadrature component at HEA was not available.

At CAP, a second site very near basin S2 (somewhat south of HEA), the in-phase arrow does not point towards the nearby basin, but rather points eastward towards the more distant White Creek Fault, indicating that at this site the fault probably plays a larger role in the resultant responses than does the basin. It could be concluded that the basin, relatively narrow near HEA and CAP, plays a minor role in the observed in-phase responses, and that the faults to the southeast could account for the observed arrow directions. Unfortunately, as was the case for HEA, the quadrature component at CAP also was not available.

At BRU, a third site near a basin boundary (southern end of basin S2), situated somewhat nearer to the basin boundary than to the Alpine Fault, the in-phase arrow in Fig. 2(a) points northwest towards the basin while the quadrature arrow in Fig. 2(b) points southeast towards the Alpine and Hope Faults. In the analogue model study of elongated conductors (simulating conductive faults) near a conductive plate (simulating an ocean, a conductive sill, or a conductive basin), Chen (1994) found that for sites roughly midway between the elongated conductor and the plate, the in-phase arrows pointed towards the plate while the quadrature arrows pointed in the opposite direction towards the elongated conductor for a range of short periods. Thus, the behaviour of the arrows at BRU (between the sedimentary basin S2 and the Alpine Fault) would seem to provide an excellent example of such oppositely directed arrows. These oppositely directed arrows, together with the significant quadrature arrow amplitude, would support the premise of the Alpine Fault being particularly conductive at depth.

At BAM, a fourth site near a basin (just west of S4), and again between a basin boundary and a fault (concealed), but in this case nearer to the basin than to the fault, the in-phase arrow, though very short, does point towards the basin. The negligible quadrature response would support the interpretation of very roughly equal and opposite quadrature responses of the shallow basin to the southeast and the concealed fault to the northwest of BAM. Since the in-phase and quadrature responses at given sites between conductors have differing period dependences (Chen, 1994), as mentioned in the previous paragraph, the net quadrature response at a specific short period could very well be zero, depending on the site location, while the in-phase arrow at that same period points towards the basin. As has been mentioned for a few other sites, the noise level in the measurements at BAM also was particularly large, and thus the arrows are not very reliable.

\subsection{Sites near multiple faults}

It is well known that, for an isolated elongated conductor in a resistive host, both the in-phase and 
quadrature induction arrows at sites on either side of the conductor point directly towards the conductor. Further, at a given period the response is maximum at roughly the conductor-host interface, and over the resistive host decreases with distance from the interface. As well, at sites over the conductor, the response decreases as a function of distance from the interface, becomes zero at the midpoint over the conductor, then with sign reversed increases as the opposite interface is approached. In the case of sites near multiple faults, however, the resultant response does not permit so simple a description, since the responses of the individual conductors may add constructively, or destructively, depending on the site location and the dimensions of the conductors. Such complex responses, expected at sites near multiple conductors, were examined by Chen (1994) in a laboratory model study of induction in parallel elongated conductors. He found that at sites between shallow and deep (large depth extent) conductors, the in-phase arrow and the quadrature arrow each reversed direction at a specific period, a period that differed for the two arrows. His results showed that induction in the deep conductor was particularly important in the quadrature response, and that depending on the relative distances from the two conductors, this arrow could very well point towards the shallow conductor at short periods, while at the same site at longer periods be reversed to point towards the deep conductor. Thus, the in-phase and quadrature arrows could very well point in opposite directions, depending on the period and the site location.

\section{i) The northern sites}

The in-phase arrows at WIR and MID (upper right in Fig. 2(a)) pointing northwest towards the Alpine and Awatere Faults respectively, would be consistent with these faults being conductive. If the two faults were equally conducive, the longer arrow at MID, compared with that at WIR, would be consistent with MID being very near the fault (Awatere) and WIR being over the fault (Alpine) near its edge. The quadrature arrow at WIR, pointing roughly eastward (oppositely directed to that of the in-phase arrow), could be accounted for by induction at depth in a conductor dipping eastward beneath WIR. The Alpine Fault is believed to extend to Moho depths, and in some regions to dip at an angle of up to $45^{\circ}$ (Grindley, 1978 ) relative to the vertical. Induction at depth in such a dipping conductor could lead to a quadrature arrow pointing south-eastward away from the fault, while the in-phase arrow, showing induction at shallow depths, could very well point north-westward towards the Alpine Fault. The quadrature arrow at MID was not available.

ii) The Lewis Pass profile sites (Karamea Bight to Pegasus Bay)

Along the Lewis Pass profile, extending from the Karamea Bight on the west coast to Pegasus Bay on the east coast, the induction arrows at BUL, REE, INA, MAR, GLY, HAE, and OMI (not including HEA, ROT, CAP, BAM discussed in Subsections 3.1 and 3.2 for sites on or near sedimentary basins S2 and S4) at most sites generally show the behaviour that could be attributed to the responses of multiple conductors. As an example, the in-phase and quadrature arrows, pointing roughly eastward at BUL, could be accounted for by the resultant responses of the S2 basin and the faults to the east (i.e. Mt. William, Lyell, White Creek Faults), if these faults were conductive. Included in the observed resultant response would be an oppositely directed component due to the fault to the northwest, if it were conductive.

Both the in-phase and quadrature arrows at REE and INA, on opposite flanks of the White Creek Fault, point in roughly opposite directions and towards the fault between the sites. The responses at INA being the smaller for both components could be accounted for by INA being the nearer to the end of the White Creek Fault, if this fault were in fact the only contributor to the responses at these sites. However, the major Alpine Fault and the basin S3 to the east would be expected to enhance the arrows pointing eastward, while attenuating the arrows pointing westward. The nearby basin S2 to the west would have the opposite effect on the observed responses at the two sites.

The in-phase and quadrature arrows at MAR, pointing in a northwesterly direction towards the nearby Awatere and Alpine Faults, and the basin S3, would be consistent with these faults and the basin being conductive. The rather moderate arrow responses, even though MAR is quite near the Awatere Fault, would tend to indicate that this fault is not particularly conductive in this region, and that the more distant Alpine Fault and the basin S3 play the major role in the responses at this site.

At HAE (the site very near a short unnamed fault, and approximately midway between the concealed 
fault to the north and the Hope Fault to the south), it would be tempting to interpret the in-phase arrow (being relatively short) as the vector sum of the responses of the short unnamed fault, the Hope Fault, and the basin S4 to the southeast, being somewhat larger than the sum of oppositely directed responses of conductors to the northwest, say those of the concealed fault and the Awatere Fault. However, with the particularly high noise level in the measurements at this site, and the quadrature arrow not available, such an interpretation may not be warranted.

At GLY, the in-phase and quadrature arrows pointing south-eastward towards the Hope Fault, would also support this fault, and the nearby unnamed branching short fault, as being conductive. Sedimentary basin S4, the concealed fault to the southeast, as well as the Clearance Fault (if conductive) to the northwest, would also contribute to the resultant responses. At OMI (very near Pegasus Bay), the in-phase and quadrature arrows seen to be pointing seaward, even though basins S4 and S5 would be expected to lead to arrows pointing inland, could be indicative of the response of an offshore conductor, a conductor which might be the extensive off shore sediments not included in the coast effect analogue model simulation. However, the marginal arrow magnitudes (approximately 0.2 ) for both components would provide rather weak support for such an interpretation.

iii) The Arthurs Pass profile sites (west coast to Banks Peninsula)

Along the Arthurs Pass profile (lower part of Figs.2(a) and 2(b)) extending from the west coast to Banks Peninsula on the east coast, the responses at sites AIK, KEL, PUN, KLO, AND, BEA, and CRA (not including NEM, BRU, SPR, and DUN discussed in Subsections 3.1 and 3.2 for sites on or near the sedimentary basins S2 and S5), would appear to be generally attributable to the local faults (if conductive), although the interpretations would seem to be even more speculative than those at sites along the Lewis Pass profile due to the multiplicity of faults of varying strike directions.

The in-phase and quadrature arrows at AIK are seen to point roughly in opposite directions. However, the quadrature arrow magnitude is rather small (less than 0.2 ) and thus will be mostly ignored in the interpretation. The in-phase arrow would be consistent with conductors to the northwest (S2 and the Hope and Alpine Faults, if conductive) providing a response component larger than that of conductors to the southeast (Kelly Fault and the concealed fault, if conductive). On the basis of model studies (Chen, 1994) of the responses at sites between elongated conductors mentioned earlier, such oppositely directed arrows (if the short quadrature arrow at this site is considered as significant) could be expected at some sites in this multi-faulted region. Depending on the location between a shallow and the deep conductor, the inphase arrow at short periods might very well point towards the distant deep conductor, and the quadrature arrow at the same period might point in the opposite direction towards the nearby shallow conductor, while at sufficiently long periods both arrows at most sites (except very near the shallow conductor) would point towards the deep conductor. Thus, the in-phase arrow at AIK, pointing towards the Alpine Fault and the nearby Hope Fault (concealed section) to the north, rather than towards the much nearer Kelly Fault, would be consistent with the Hope and Alpine Faults being conductive, and providing a response component larger than any provided by the Kelly Fault, or by the short concealed fault to the east.

The roughly oppositely directed arrows at KEL, a site roughly on the Kelly Fault, would not be inconsistent with the interpretation of the in-phase arrow at AIK, if KEL is in fact on the west flank of the Kelly Fault, and both this fault and the concealed fault to the east were particularly conductive at shallow depths, while the Hope and Alpine Faults to the northwest were conductive over a large depth range.

The arrow directions at KLO and PUN could also be consistent with the resultant response expected of multiple conductors. In the case of KLO, the nearby concealed fault and the more distant faults (Kelly and Alpine) to the northwest, providing a larger resultant response component than the faults to the southeast. In the case of PUN, on the opposite side of the concealed fault, the quadrature arrow pointing roughly in the opposite direction, would be consistent with the concealed fault between the two sites being particularly conductive. However, with the noise level in the measurements at PUN being rather large, this interpretation, for the quadrature arrow pointing towards the fault, and the in-phase arrow response being essentially zero, would not be reliable.

Since BEA, AND and CRA are situated in a region of closely spaced and interconnected faults, the 
interpretations of the difference arrows here would be expected to be rather speculative. However, the inphase arrow at BEA (roughly $5 \mathrm{~km}$ east of KLO), pointing to the northwest, is consistent with the earlier premise that the concealed fault (near KLO) and the Kelly and Alpine Faults, all to the northwest, are conductive. The quadrature response at BEA is too small to warrant comment, but as explained earlier, for the case of multiple conductors, the resultant quadrature response could very well be zero while the in-phase response is significant, depending on the site location and the period. The field measurements at AND were known to be in error, and thus the difference arrows are not shown for this site. At CRA, the in-phase arrow direction, being roughly parallel to the NE-SW trending local faults, could be accounted for by the responses of these multiple faults roughly cancelling, and the observed arrow then being the response of some unknown conductor to the northwest. However, both in-phase and quadrature responses for a broad range of periods would be required to confirm any premise of such a conductor in this region.

\section{Conclusions}

The $10 \mathrm{~min}$ period in-phase and quadrature difference arrows at some of the 26 sites in the Southern Alps region appear to be attributable to the induction responses of conductive sedimentary basins and faults. At several sites, the directions and magnitudes of both the in-phase and quadrature arrows would support a premise that the Alpine, Hope, and Kelly Faults, tend to be conductive at some depths, and that some of the older concealed faults are particularly conductive. Measurements for a much more detailed period range would be required to permit a fuller interpretation of difference arrows in terms of such anomalous conductors in this complex Southern Alps region.

The financial support through an NSERC Grant (H.W.D) and a University of Victoria Faculty Research Grant (H.W.D.) is gratefully acknowledged.

\section{REFERENCES}

Bapat, V. J., J. Segawa, Y. Honkura, and P. Tarits, Numerical estimation of the sea effect on the distribution of induction arrows in the Japanese island arc, Phys. Earth Planet. Inter., 81, 215-229, 1993.

Bromley, C. J., Geomagnetic depth sounding and magneto-telluric survey of the Southern Alps. M.Sc. Thesis, Victoria University of Wellington, Wellington, New Zealand, 1979.

Chamalaun, F. H. and J. D. McKnight, A New Zealand wide magnetometer array study, J. Geomag., 45, 741-759, 1993.

Chen, J., Electromagnetic induction in the New Zealand region. Ph.D Thesis, University of Victoria, Victoria, Canada, 1994.

Chen, J., H. W. Dosso, and M. Ingham, Electromagnetic induction in New Zealand: analogue model and field results, Phys. Earth Planet. Inter., 62, 257-270, 1990.

Chen, J., H. W. Dosso, and M. Ingham, Electromagnetic induction in the New Zealand South Island, Phys. Earth Planet. Inter., 81, 253-260, 1993.

Dosso, H. W. and Z. Meng, The coast effect response in geomagnetic field measurements, Phys. Earth Planet. Inter., 70, 39-56, 1992.

Grindley, G. W., Alpine schist belt-Haast to Wanganui rivers, south Westland, in The Geology of New Zealand, Volume 1, edited by R. P. Suggate, Government Printer, Wellington, New Zealand, 1978.

Kang, S., H. W. Dosso, and S. O. Ogunade, Electromagnetic induction in South-west Nigeria: analogue model and field results, J. Geomag. Geoelectr., 45, 805-816, 1993.

Lilley, F. E. M. and B. R. Arora, The sign convention for quadrature Parkinson arrows in geomagnetic induction studies, Rev. Geophys. Space Phys., 20, 513-518, 1982.

Meng, Z. and H. W. Dosso, An analogue model study of EM induction in the Japan-Korea-China region, Phys. Earth Planet. Inter., 62, 246-256, 1990.

Weaver, J. T. and A. K. Agarwal, Is addition of induction vectors meaningful?, Phys. Earth Planet. Inter., 65, $267-275,1991$.

Wolf, D., Inductive coupling between idealized conductors and its significance for the geomagnetic coast effect, J. Geophys., 52 , 22-33, 1983. 